\title{
SKATING ON SLIPPERY ICE
}

I T.H. Oosterkamp, T. Boudewijn and J.M.J. van Leeuwen - DOI: https://doi.org/10.1051/epn/2019104 - LION, Universiteit Leiden, Niels Bohrweg 2 - 2300 RA Leiden - The Netherlands.

Skating has been popular for centuries but is still poorly understood. Is it sufficient that the surface of ice is wet in order to explain why skating is possible for a wide range of temperatures, velocities and types of skates? Or do we need a layer of water formed between skate and ice by the frictional heat? Here we discuss the physics of the water layer and its implications at large velocities as occurring in speed skating.

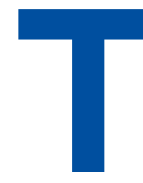

he friction of a skate on ice is orders of magnitude smaller than the friction between steel and another solid. So one wonders what exceptional property of ice leads to this low friction. Ice has the rare property that the solid phase is less dense than the liquid phase: ice floats on water. This property has a profound impact on our habitat. Would life be possible if the polar ice would sink and accumulate at the bottom of the ocean? Skating is a minor beneficiary of this property, as canals freeze on top and not at the bottom. So, after a few nights of frost, an ice layer develops sufficiently thick to carry a human body. The fact that the

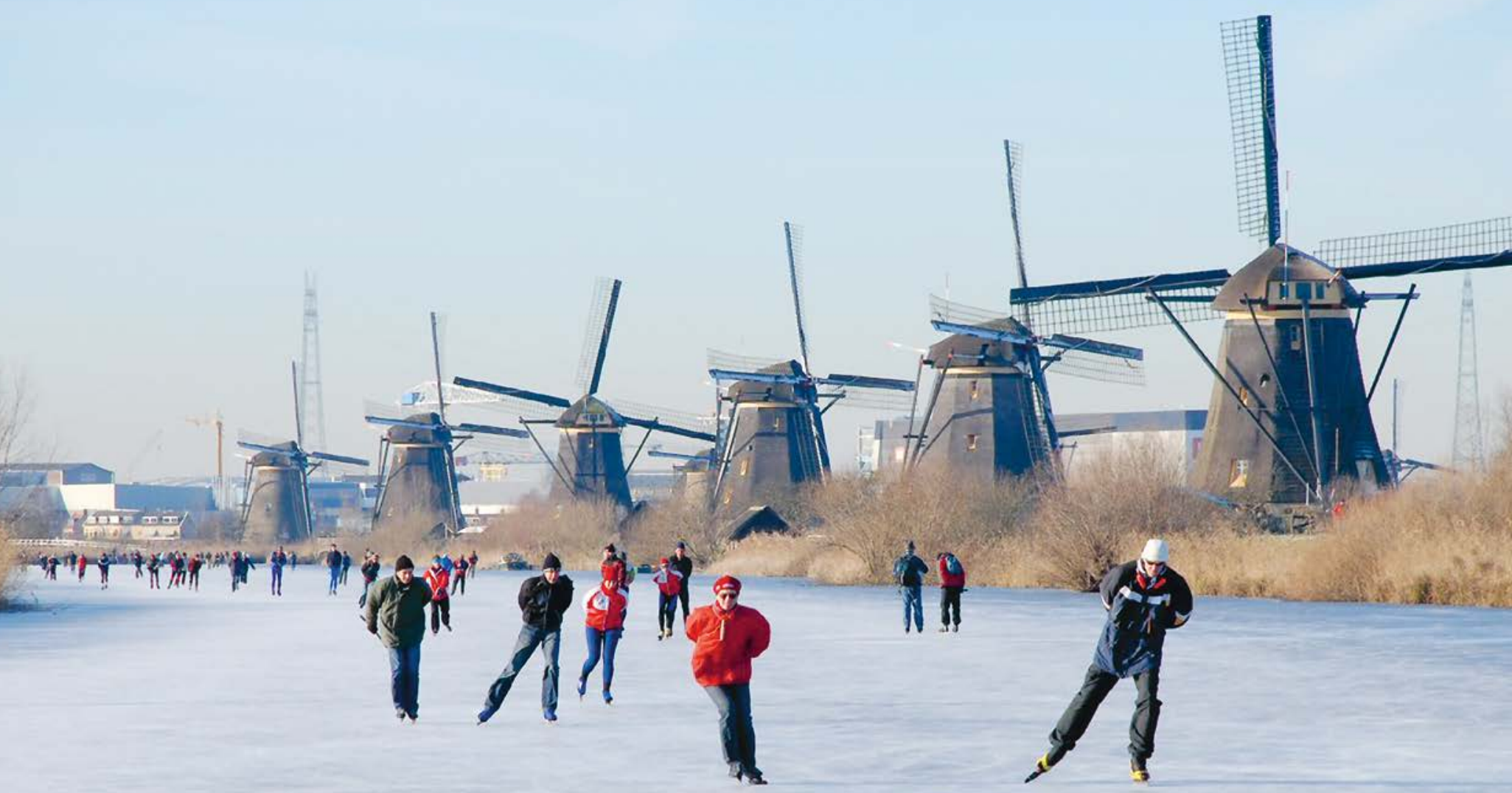


volume change from ice to water is negative has another implication through the Clausius-Clapeyron relation

$$
\frac{d P}{d T}=\frac{L_{h}}{T \Delta v} .
$$

Here $\Delta v$ is the (negative) volume change from ice to water, $T$ the absolute temperature and $L_{\mathrm{h}}=0.33 \cdot 10^{6} \mathrm{~J} /$ $\mathrm{kg}$ the latent heat of melting. $d P / d T$ is the derivative of the melting line and, as $\Delta v<0$, the melting temperature decreases with increasing pressure. This feature has led to the still popular, though wrong, explanation of skating: due to the pressure exerted by the weight of the skater, the ice underneath the skate melts and the skate glides on this layer of water. The lowering of the melting temperature under pressure was once demonstrated in high schools with a steel cable, melting itself through a block of ice under the pressure of a load. The spectacular aspect is that above the steel cable the water regelates, such that after the steel cable has melted itself through the block of ice, the two parts still form one solid block of ice!

The simplest objection against this explanation is that skaters, leading to a pressure of some $15 \mathrm{MPa}$, give only a lowering of 1 degree centigrade, while skating is perfectly possible at 10 degrees below freezing. Apart from this argument one would have to explain the origin of the heat necessary to melt a sufficient layer of water. The steel cable gives a clue. The regelation of the ice above the cable, provides the heat needed to melt ice underneath the cable. It works due to the high heat conductivity of steel. A nylon cable with equal pressure does not melt the ice.

Faraday [1] already suggested that the surface of ice is wet. This leads to another explanation of skating, based on the motto: slippery when wet. Careful experiments combined with molecular dynamics calculations $[2,3,4]$ have demonstrated the existence of a surface with a layer of very mobile molecules for temperatures above $-30^{\circ} \mathrm{C}$. The strong similarity in the behaviour of the surface mobility and the friction as function of the temperature, prompted the authors to see this layer as the key to the slipperiness of ice. Such a surface certainly contributes to lowering the friction, but the question is whether it is sufficient for skaters of a weight of $75 \mathrm{~kg}$ with a speed of $15 \mathrm{~m} / \mathrm{s}$. Then the ice is touched for about a millisecond and one has to show that surface mobility can keep up with this fast process.

The more standard approach links the heat of friction to the formation of a thin layer of water between the skate and the ice. Such a layer has never been demonstrated experimentally. Efforts to detect the layer are underway [5], but are difficult since the water layer is calculated to be only $1 \mu \mathrm{m}$ thick. So up till now, the water layer features only through its implications, following from the hydrodynamics and the heat balance of the water layer. In this article we focus on the quantitative analysis of the pressure and temperature distribution [6] in the layer of
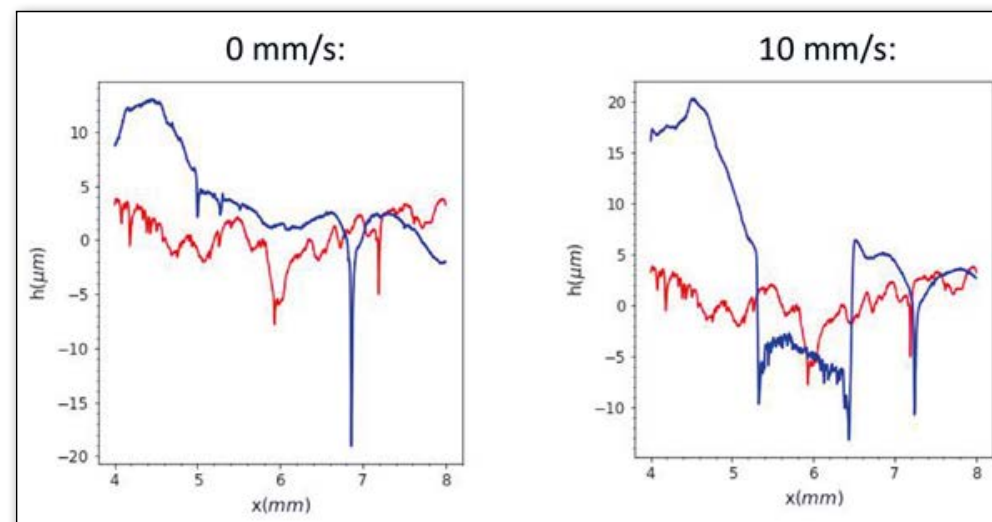

$30 \mathrm{~mm} / \mathrm{s}$ :

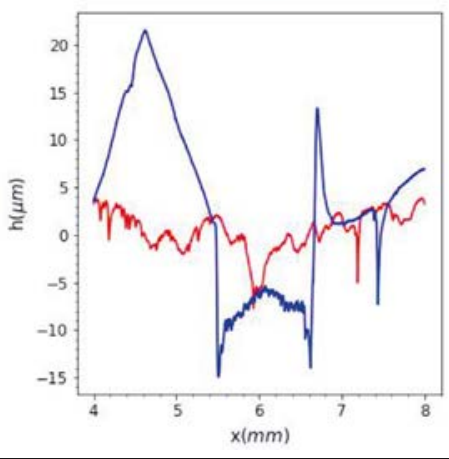

$100 \mathrm{~mm} / \mathrm{s}$ :

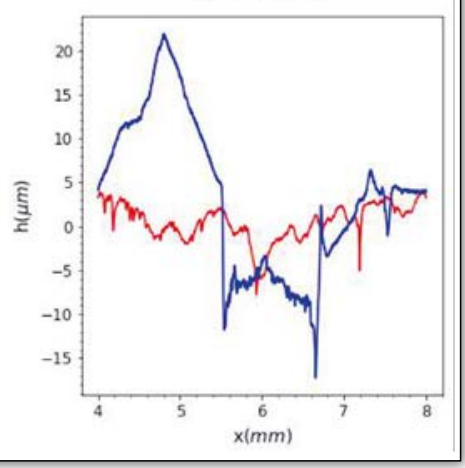

water, which is created by and which controls in part the friction of the skate with the ice.

Not only the thickness of the water layer is important, but also the depth of the indentation that the skate causes in the ice. Indentation is essential for skating, since skating is not only a matter of gliding, but also of pushing oneself forward. This requires sharp edges on the skates. Figure-, hockey- and speed skaters know that sharp edges are extremely important for a good performance. As we are most interested in skating at high velocity, we restrict ourselves to speed skates, having a rectangular shape in the transverse direction with a width $w=1.1 \mathrm{~mm}$ and a radius of curvature $R=22 \mathrm{~m}$ in the longitudinal direction.

Sharp edges always lead to a plastic deformation of the ice and therefore the hardness of ice plays a central role. The hardness is the pressure above which deformations are plastic and no longer elastic. Before we embark on the physics of skating we discuss briefly the hydrodynamics of a sheared thin layer of water.

\section{The hydrodynamic properties of a layer of water under shear}

Consider (box 1) a layer of water of thickness $h$, which on top moves with the speed $V$ of the skate and which is at the bottom at rest, such that in the vertical direction a velocity gradient $V / h$ exists. As the layer is thin, the lubrication approximation applies and the gradient causes at the top a friction force $F_{\mathrm{w}}=\eta V / h$ per area, with $\eta=1.7 \cdot 10^{-3}$ Pas. So the friction of the skate is $F_{\mathrm{w}} w l$, where $w$ is the width of the skate and $l$ the contact length. An estimate, to be articulated later, gives the contact length from the $\triangle$ FIG. 1: Indentations for static and dynamic deformations. 


\section{BOX 1: FRICTION AND PRESSURE IN THE WATER LAYER}

Fig. 3 shows the velocity profile in a thin layer of water of thickness $h$ moving at the top with velocity $V$ and at rest on the bottom. The velocity in the longitudinal $x$-direction is given by

$$
v_{x}=V z / h \text {, }
$$

with $z$ the perpendicular direction. In order to keep the water moving at the top one has to apply a shear force

$$
F_{\mathrm{fr}}=\eta\left(\frac{V}{h}\right) w l,
$$

with $V / h$ the velocity gradient, $w$ the width in the transverse $\gamma$-direction and $/$ the contact length.

There is also a velocity profile $v_{y}$ in the transverse direction

$$
v_{y} \sim y z(h-z),
$$

since the water is squeezed out sideways. $v_{y}$ vanishes at the top and the bottom of the layer. The pressure needed for this transverse flow is of the form

$$
p-p_{\mathrm{h}} \sim \eta\left(w^{2} / 4-y^{2}\right) .
$$

It is a parabola with the top in the middle $y=0$. Eqns. (13) and (14) satisfy the hydrodynamic equation $\nabla p=\eta \Delta v$.

force balance: the weight of the skater must be balanced by the product of the pressure times the contact area

$$
\mathrm{Mg}=\mathrm{pwl} .
$$

Here $M$ is the mass of the skater and $p$ the pressure. We will argue that the deformations of ice due to skating are mostly plastic. Evidence for plastic deformation is the visible trail that a skater leaves behind on virgin ice and the fact that a skating rink has to be regularly mopped up from the ice debris. So the hardness $p_{\mathrm{h}}$ is a reasonable figure to get an order of magnitude estimate. Pourier et al. [7] give for the hardness the formula

$$
p_{\mathrm{h}}=14.7-0.6\left(T-T_{\mathrm{m}}\right) \text {, }
$$

with $p_{\mathrm{h}}$ in MPa and the temperature difference $T-T_{\mathrm{m}}$ in centigrade. This expression is open for critique $[8,9]$, since other measurements give substantial lower values and the method of measuring the hardness by dropping steel balls is somewhat disputable. We take for the calculations $p_{\mathrm{h}}=10 \mathrm{MPa}$ and a skater of $72 \mathrm{~kg}$. Then the contact length $l=6.4 \mathrm{~cm}$. That in turn gives for a layer of $10 \mathrm{~nm}$, a friction force of $122 \mathrm{~N}$. This is an order of magnitude higher than the measured value by de Koning et al. [10], who find for a skater of $72 \mathrm{~kg}$ frictions in the range of $3.8 \mathrm{~N}$ for the straights and $4.9 \mathrm{~N}$ for the curves. That a straight stroke has less friction than a curved stroke, indicates that an upright skate has less friction than a skate in a slant position. The analysis of an upright skate is easier than that of a slant skate and therefore we restrict ourselves to the former case.
As the friction is an order of magnitude too large for a layer of water of $10 \mathrm{~nm}$, it is a problem to understand that shrinking the layer to $1 \mathrm{~nm}$ would bring the friction in the right ball park.

\section{A stable layer of water}

Friction produces heat at the interface between skate and ice. But is the heat sufficient to melt a layer of ice into water, forming the interface between skate and ice? The answer is positive, since the layer is self-establishing. If the layer gets thinner by a fluctuation, the heat generation increases making the layer thicker. In the same way a fluctuation enlarging the layer is damped out by lowering the friction and the heat generation. This stability makes skating robust against irregularities in the ice surface. One has to show however that the generated heat is indeed available for melting and does not leak away e.g. to the skate instead of to the ice. In box 2 we make up the heat balance between the generation of heat and the thickness of the water layer. It results in the layer equation, describing the thickness $h(x)$ as function of the position $x$ along the skate.

$$
-\frac{d h(x)}{d x}=\frac{k}{h(x)} .
$$

Here $k$ is a parameter of the dimension of a length

$$
k=\frac{\eta V}{2 \rho L_{\mathrm{h}}},
$$

with $\rho$ the density of ice $\rho=962 \mathrm{~kg} / \mathrm{m}^{3}$ and $L_{\mathrm{h}}$ the latent heat of melting $L_{\mathrm{h}}=0.33 \mathrm{MJ} / \mathrm{kg}$. $k$ is quite small: $\mathrm{k}=2.8 \cdot 10^{-11} \mathrm{~m}$, for $V=10 \mathrm{~m} / \mathrm{s}$. The solution of Eq. (4) reads

$$
\mathrm{h}(\mathrm{x})=\sqrt{2 k(l-x)},
$$

with $l$ again the contact length as integration constant. So the layer vanishes at the tip of the skate $x=l$ and has it largest thickness in the middle at $x=0$. With $l=6.4 \mathrm{~cm}$ we find the small value $h(0)=\sqrt{2 k l}=1.9 \mu \mathrm{m}$ This makes the experimental demonstration of the water layer difficult. In the derivation we have assumed that the generated heat streams in equal proportions towards the ice and the skate, which is the case when the skate has the temperature of the ice. This is accounted for by the 2 in the denominator of Eq. (5) for $k$. As it is unlikely that the skate gets colder than the ice, $1 / 2$ is a lower limit.

An important consequence of the solution (6) is that it leads to the friction

$$
F_{\mathrm{w}}=\eta \mathrm{w} \int_{0}^{l} d x \frac{V}{h(x)}=2 \eta w V\left(\frac{l}{2 k}\right)^{1 / 2}=2 w \sqrt{\eta \rho L_{\mathrm{h}} V l} .
$$

That the friction depends on the skate velocity as $\sqrt{V}$ is surprising. Approaching $\mathrm{V} \rightarrow 0$ the layer vanishes but the friction also vanishes! For $V=10 \mathrm{~m} / \mathrm{s}$ the friction force $F_{\mathrm{w}}=1.3 \mathrm{~N}$. This value is substantially lower than the measured $4 \mathrm{~N}$ by de Koning et al. [10]. Clearly we must have missed a few loss mechanisms. We list a number of the possibilities. 
- The water does not stay underneath the ice, but is squeezed out sideways. One can account for this effect by computing the pressure distribution in the water layer. The pressure varies as a parabola with the top in the centre and the lowest points at the edges, where the water escapes from the layer.

- In box 2 we have assumed that the upper surface of the layer is flat, while it is curved by the curvature of the skate. - By the melting, the temperature of the surface of the ice is suddenly raised to the melting temperature (to be precise to the melting temperature at the pressure in the layer). This heat shock causes a gradient inside the ice by which heat also leaks away inside the ice.

- We calculated the friction for an upright skate, while skating happens mostly at an angle, even for the straight strokes. The measurements of de Koning et al. [10] indicate indeed that the upright position has the least friction.

- Ice and skates are not perfectly smooth etc.

The combined effect on $F_{w}$ of the first two items is a substantial increase of the friction, as it shrinks the water layer. However, if we consider the pressure distribution, we have to include also the influence of the pressure on contact length and the indentation of a skate in ice. Most importantly of all omissions is that we have ignored the friction due to motion of this indentation: the skate does not only glide over the water, but also has to plough its way through the ice.

\section{Ploughing}

The indentation $d(x)$ is a sphere segment, approximately given by

$$
d(x) \approx d-\frac{x^{2}}{2 R}, \rightarrow d \approx \frac{l^{2}}{2 R} .
$$

It is maximal in the middle of the skate $d=d(0)$ and for $l=6.4 \mathrm{~cm}$ it equals $94 \mu \mathrm{m}$. A simple expression for the ploughing friction is [11]

$$
F_{\mathrm{pl}}=p_{\mathrm{h}} w d .
$$

$w d$ is the cross-section of the furrow that the skate ploughs against the hardness as counter pressure. For the skating parameters we find $F_{\mathrm{pl}}=1.0 \mathrm{~N}$. Addition of $F_{\mathrm{w}}$ from Eq. (7) to $F_{\mathrm{pl}}$ then leads to the total friction force.

That the skate really ploughs through the ice is demonstrated by the laboratory experiment [13], in which a skate is pushed over a strip of ice. A sensitive roughness tester scans the height of the ice surface. In Fig. 1 we show the results. The red curves give, as reference, the surface in a run of the tester perpendicular to the skate, before the skate has touched the ice. Pushing the skate into the ice with a force of $500 \mathrm{~N}$ and removing it, leaves the ice in a state which is tested again. The blue curve in the upper left corner shows a minor difference between the surface before and after the move. This indicates that the deformation is mostly elastic. In contrast when the skate is moved with a velocity, the skate leaves a clear indentation behind, showing that the deformation is plastic and that the skate leaves a furrow behind. The difference between static and dynamic behaviour is simply explained by the fact that a static deformation has twice the contact length of a dynamic deformation. So the counter pressure of the ice in the dynamic case, needs to be twice that of the static case. Using the values of the hardness for $T=-10^{\circ} \mathrm{C}$, one finds indeed that in the dynamic case the pressure exceeds the hardness for $500 \mathrm{~N}$. This makes the indentation plastic.

However, we should realize that in the dynamic case the skate does not directly touch the ice. It is not the skate, but the pressure in the water layer that pushes the ice down. Moreover the pressure in the water layer exceeds the hardness, amongst others in order to squeeze out the water sideways. And for speed skating the pressure must be such that the ice is pushed down with a certain velocity. For a velocity of $10 \mathrm{~m} / \mathrm{s}$ the ice at the tip of the skate has to give way downwards at a rate of $1 \mathrm{~cm} / \mathrm{s}$. Therefore we need a sort of dynamic hardness that gives the rate $v_{\text {ind }}$ at which the ice indents. We propose for this rate the following relation, inspired by the Bingham solid, [6]

$$
v_{\text {ind }}=\gamma\left(p-p_{\mathrm{h}}\right) .
$$

Below $p_{\mathrm{h}}$ the deformation is elastic, above $p_{\mathrm{h}}$ the ice starts to flow with the velocity $v_{\text {ind }}$. We have no measurement of $\gamma$, only an estimate, coming from shear flow of ice. We use $\gamma p_{\mathrm{h}}=1 \mathrm{~mm} / \mathrm{s}$ for the computation.

The above formula is an interpolation between two recent theories on skating. Lozowski and Szilder [11] implicitly assume that the pressure in the water later always equals the (static) hardness. This comes down to a value $\gamma=\infty$. On the other hand Le Berre and Pomeau [12] ignore the rate of indentation, which boils down to a value $\gamma=0$. Now the indentation rate is dictated by the shape of the skate. It behaves as $v_{\text {ind }}=V x / R$, with $x / R$ the slope of $d(x) . v_{\text {ind }}$ is maximal at the tip of the skate $(1 \mathrm{~cm} / \mathrm{s}$ at $V=10 \mathrm{~m} / \mathrm{s})$ and zero in the middle. So Eq. (10) gives the pressure inside the water layer needed to realize this velocity. That would imply that the pressure at the tip, is 11 times the static hardness for $V=10 \mathrm{~m} / \mathrm{s}$ ! Such a pressure increase shortens the contact length,

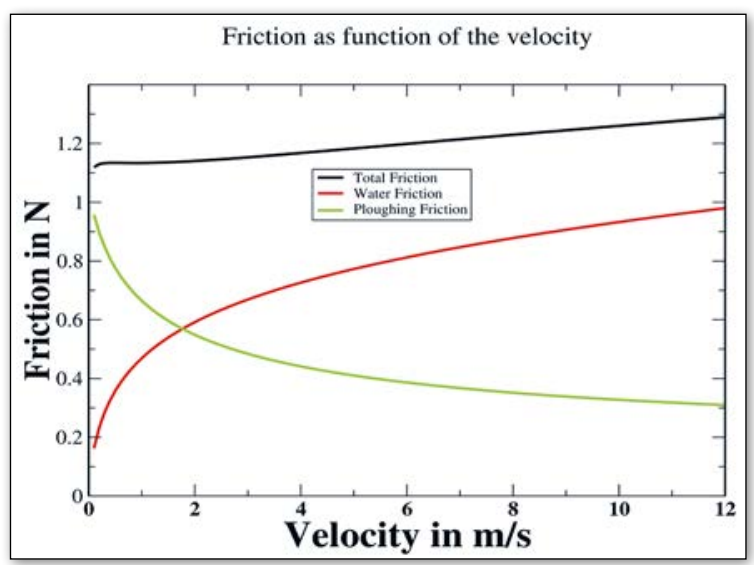

4 FIG. 2:

Total friction as function of the velocity $\mathrm{V}$

which has a twofold consequence. 
$\triangle$ FIG. 3: A thin layer of water between the skate and the ice.

- A shorter contact length $l$ yields a smaller indentation depth $\mathrm{d}=l^{2} / 2 R$. If the average pressure is twice the static hardness, the contact length is reduced by a factor 2 and the depth $d$ by a factor 4 . So the ploughing product $p w d$ is reduced by a factor 2 . The counterintuitive result, that ploughing faster meets less resistance, is a form of aquaplaning: the skate is lifted and ploughs less deep through the ice.

- A shorter contact length reduces also the friction $F_{\mathrm{w}}$ as ice and water get a smaller surface of contact. This effect is substantial and undoes for the greater part the rise in friction due to the thinner water layer.

If we take all facets into account, we get the following picture for the total friction as function of the velocity $V$ (see Fig. 2).

The rise of the resistance due to the friction in the water layer is largely compensated by the reduction of the ploughing resistance. As result we get a weakly rising

\section{BOX 2: THE HEAT BALANCE}

V FIG. 4: Heat generation and melting in a volume v

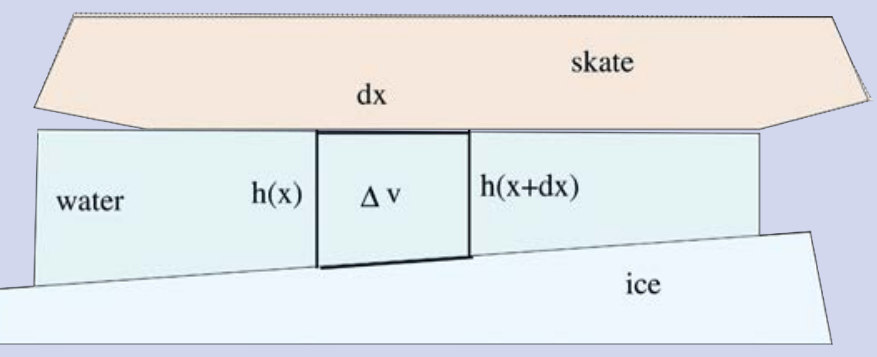

The rate of heat generation in a volume $\Delta v=h(x) w d x$ reads

$$
\frac{d H(x)}{d x}=\eta \frac{V^{2}}{h^{2}(x)} \Delta v=\frac{V^{2}}{h(x)} w d x .
$$

This leads to an increase of the water volume

$$
\frac{d \Delta v}{d x}=\frac{d H(x)}{d t} \frac{1}{2 \rho L_{\mathrm{h}}}
$$

assuming that only half of the heat flows towards the ice and the other half flows towards the skate. On the other hand the volume increases via $d x=-V d t$ as

$$
\frac{d \Delta v}{d x}=w \frac{d H(x)}{d t} d x=-w \frac{d h}{d x} V d x
$$

Equating these two expressions gives the layer equation (5) in the text. friction as function of the velocity, which makes skating at high speeds possible: the world record is $93 \mathrm{~km} / \mathrm{hr}$. The computed values have a large margin of uncertainty, mainly due to the uncertainty in $p_{\mathrm{h}}$ and $\gamma$. Fortunately the total friction varies only mildly with the value of $\gamma$. An increase of a factor 100 in $\gamma$ gives about a factor 2 increase in the total friction. Nevertheless a test of Eq. (10) is more than welcome!
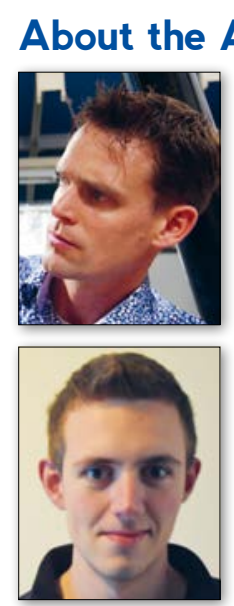

Thom Boudewijn is Master Student in experimental phyics at the University of Leiden. His Masters Project is a laboratory experiment of a skate gliding over a thin layer of ice, with the aim of measuring the friction and indentation of the skate in ice. J.M.J. van Leeuwen is emeritus professor in theoretical physics at the Lorentz-Institute of the University of Leiden. Apart from his interest in skating he is concerned with problems in Statistical Physics.

\section{References}

[1] M. Faraday, Experimental Researches in Chemistry and Physics, Taylor and Francis, London (1859) p. 372.

[2] B. Weber, Y. Nagata, S. Ketzetzi, F. Tang, W. J. Smit, H. J. Bakker, E. H. G. Backus, M. Bonn and D. Bonn, J. Phys. Chem. Lett. 911 , 2838 (2018)

[3] W. J. Smit and H. J. Bakker, Angew. Chem. 129, 15746 (2017)

[4] W. J. Smit, F. Tang, M. A. Sánchez, E. H. G. Backus, L. Xu, T. Hasegawa, M. Bonn, H. J. Bakker, Y. Nagata, Phys. Rev. Lett. 119, 133003 (2017)

[5] T. H. A. van der Reep, Masters Thesis (2014) Univ. Leiden.

[6] J. M. J. van Leeuwen, SciPost Phys. 3, 042 (2017).

[7] L. Pourier, R. I. Thompson, E. P. Lozowski, S. Maw and D. J. Stefanyshyn, $21^{\text {st }}$ Int. Offshore and Polar Eng. Conf. (2011) Maui, ISOPE, 31071.

[8] B. Weber, PhD. thesis (2017) Univ. Amsterdam.

[9] A. Penny, E. P. Lozowski, T. Forest, C. Fong, C. Maw, P. Montgomery and N. Sinha, Physics and Chemistry of Ice (2007) 495, W. F. Kuhn, editor, Roy. Soc. Chem.

[10] J. J. de Koning, G. de Groot and G. J. van Ingen Schenau, J. Biomechanics 25, 565 (1992).

[11] E. P. Lozowski and K. Szilder, Int. Journ. of Offshore and Polar Engineering 23, 04 (2013).

[12] M. Le Berre and Y. Pomeau, Int. Journ. of Non-linear Mech. 75, 77 (2015).

[13] T. Boudewijn, Masters Thesis, (2019) Leiden University. 